\title{
A Contrastive Research on the Definitions and Categories of Chinese and English Idioms
}

\author{
You Wang ${ }^{1,2^{*}}$ \\ ${ }^{1}$ School of Foreign Languages, Jianghan University \\ ${ }^{2}$ Research Center for Language and Language Education, Central China Normal University \\ *You Wang. Email: wangyou1018@163.com
}

\begin{abstract}
Chinese and English belong to Sino-Tibetan language family and Indo-European language family respectively. There are according differences between the idioms of the two languages. The definition and classification of Chinese and English idioms have always been the focus of debate in academic circles. This study, through the comparison of definitions and categories between Chinese and English idioms, comes to the conclusion that the classification of Chinese idioms emphasizes the semantic and pragmatic features of the targets. Chinese idioms can be roughly divided into idioms, proverbs, common sayings, two-part allegorical sayings and aphorisms. On the contrary, the classification of English idioms focuses on syntactic structure. Thus English idioms are divided into eight categories: sentence idioms, semi-sentence idioms, verbal phrases, prepositional phrases, nominal idioms, adjective idioms, paired words and fixed similes.
\end{abstract}

Keywords: Chinese idioms, English idioms, definition, classification, comparison between Chinese and English

\section{INTRODUCTION}

The contrastive study of Chinese and English idioms in recent years has undergone rapid development, laying stress on the field of cognition and translation. However, these studies have failed to focus on the source of idioms, that is, the definition and classification of them [1]. Chinese and English languages belong to different language families, thus inevitable differences in the definition and classification of idioms. Therefore, the research of Chinese and English idioms should be done on the basis of the differences. For example, are the definitions of Chinese and English idioms identical? Is their classification basis the same? This essay intends to start from the definition, and then to discuss the differences in the classification criteria between Chinese and English idioms. It is committed to making contribution to the fundamental work of contrastive study of Chinese and English idioms.

\section{A CONTRASTIVE RESEARCH ON THE DEFINITION OF CHINESE AND ENGLISH IDIOMS}

As a loanword derived from Russian, the term "idiom" in Chinese refers to "the sum of all fixed phrases" in a language. In addition, it is also "a discipline that studies fixed phrases, namely "idiomology" [2]. Flourished in the 1950s, idiomology is a branch of linguistics. It is still young compared with other disciplines. The early research on idioms in China centered on the characteristics, origin and application of idioms. Most researchers in that period, under the influence of the idiom research in the former Soviet Union, thought that idioms were "special words" which should fall into the category of lexicology. This idea agrees with the one that studies the corresponding subordinate categories in the framework of semantics, pragmatics and sociolinguistics. After the reform and opening up, all kinds of academic research is in the ascendant, and the study of idioms is thereupon constantly deepening and developing. Scholars summarize all kinds of research results and conceptualize the term "idiom". Ci hai provides the following interpretation for "idiom": "Idioms are 
commonly used and stereotyped phrases or sentences, which have a long history. They are widely used and very expressive." This definition fully extends the extension of "idiom" on the basis of the original definition. As a result, the idiom includes both "phrase" and "sentence". The interpretation also shows that "idiom" is a concept of "species" at the species level. It is a superordinate in the semantic field, with all the commonly used and stereotyped phrases or sentences as its hyponyms.

Yao Xiyuan [3] also thinks that "idiom" is a "species", which covers all in species status and definition domain of "idioms". The reasons include: 1 . In the view of denotation, idiom is a concept of "species". It is a linguistic term translated from Russian. "Idiom" in Russian Encyclopedia Dictionary published by the former Soviet Union is interpreted as "the sum of the idiom units of a language". It is also believed in many linguistic works in western countries that idiom includes idioms, aphorisms, proverbs and so on. 2. Many linguists also believe that "idiom" belongs to the concept of "species". For example, Mr. Ma Guofan has a clear conclusion on the types of idioms, "There was no clear definition of idiom in the past, which made the morphological features difficult to define. After the term was translated from Russian, it is used to refer to the total of various forms of fixed phrases or word groups. Idioms, aphorisms, proverbs, beautiful lines, etc. belong to the category of idioms." In addition to Mr. Ma Guofan, the concept of idiom as "species" has also been recognized by scholars such as Wu Zhankun and Liu Shuxin. It is a point of view well accepted by Chinese linguists. Consequently, "idiom", as a set of established system, should be regarded as "species". 3. The formation of a concept comes from people's language practice. Likewise, the formation of various genus concepts in idiom is also the product of people's long-term language practice. It is not done at once, but gradually fixed in the language system. Each concept under the idiom has its own special denotation. Moreover, the language phenomena of its reference are also characteristic and different. If we use a certain concept of "genus" under the idiom to assume the role of the concept of "species", it is not in line with the linguistic facts, nor is it scientific. The term "idiom" is not a specific term, but is used to refer to all kinds of fixed idioms. Therefore, taking idiom as a concept of "species" is easy to be accepted.

Linguists at home and abroad have studied English idioms for more than half a century. However, they have not reached a complete consensus on the definitions and characteristics of English idioms. Linguists and lexicographers both express their own opinions and have their own reasons. Some researchers believe that English idioms should be commonly used phrases and word groups. Some scholars define them as the idiomatic collocation of words. They include not only phrases but also sentences. However, others have extended the scope of English idioms to the combination of words and various combinations other than words, such as the usage of words and so on. Many scholars propose to take the particularity and indivisibility of meaning as the criteria for determining idioms from the perspective of semantics. However, other scholars take the structural form of idioms, that is, whether the word order is fixed or not as the criteria. The reason of why there are so many different voices in the definition of English idioms is the feature of English language itself: As a unique language expression of the Anglo-Saxon people, English idioms have broad connotations. They are complex and diverse in type, structure, meaning and function. It is really difficult to give a perfect definition of English idioms.

Famous scholar Chen Bosong, in his book Introduction to English Idioms lists 11 interpretations of idioms in natural languages given by linguists and lexicographers at home and abroad. Among them four interpretations can be adopted for reference.

1) "An idiom is an accepted phrase, construction, or expression contrary to the usual patterns of the language or having a meaning different from the literal."

2) "An idiom is a succession of words whose meaning must be learned as whole."

3) "An idiom is a fixed group of words with a special different meaning from the meanings of the separate words."

4) "An idiom is made up of two or more words. They are used to express a concept, functionally equivalent to a word, and they serve as a sentence component syntactically. "

The definition given by Chen Bosong in his book is "stereotyped, integrated idiomatic phrases or short sentences". Most scholars have the same understanding of "idiomatic, stereotyping and integrated" though it is hard to define English idioms. Differences mainly lie in the structural form and semantic relations of idioms.

The preface of Collins Cobuild Dictionary of Idioms [4] gives a very detailed explanation of "idiom":

An idiom is a special kind of phrase. It is a group of words which have a different meaning when used together from the one it would have if the meaning of each word were taken individually. If you do not know that the words have a special meaning together, you may well misinterpret what someone is saying, or be puzzled by why they are saying something that is untrue or irrelevant. For example, learners might not recognize the idioms bite someone's head off and out in the cold. They would then have problems understanding remarks such as "Don't bite my head off just because you're bad tempered" and "They were going to play together and that left me out in the cold, you know". 


\section{A CONTRASTIVE RESEARCH ON THE CATEGORIES OF CHINESE AND ENGLISH IDIOMS}

The classification of idioms is different in modern Chinese academia. Seven viewpoints can be roughly seen:

1) Idioms, are stereotyped phrases or sentences in a language. That is, the relatively fixed idiomatic expressions formed by the long-term use of various nationalities, and in the wide spread of the language.

2) Wang Dechun in his Study of Lexicology (1983) divides idioms into five categories: 1) idioms, 2) proverbs, 3) maxims and aphorisms, 4) two-part allegorical sayings, 5) common sayings and locutions.

3) Hu Yushu in Modern Chinese (1981) believed that idioms include idioms, locutions, two-part allegorical sayings, proverbs and aphorisms. Sun Weizhang in Study of Chinese Proverbs (1989) and the Dictionary of Chinese idioms edited by Wen Duanzheng (1996) share the same view.

4) Summary of Modern Chinese Vocabulary by Wu Zhankun and Wang Qin (1983): idioms include idioms, locutions, two-part allegorical sayings, and proverbs.

5) Fu Huaiqing in Modern Chinese Vocabulary (1985) indicates: idioms include idioms, proverbs, and common sayings and two-part allegorical sayings.

6) Modern Chinese (1997), edited by Huang Burong and Liao Xudong says that idioms include idioms locutions, two-part allegorical sayings. Scholars who hold this definition and classification define idioms as fixed phrases that are commonly used, which is a special lexical unit.

7) Wang Qin's Reserach on Chinese idioms (2006): idioms include idioms, two-part allegorical sayings, locutions, common sayings and proverbs.

The following may discuss the nature and research scope of Chinese idioms, proverbs, common sayings, two-part allegorical sayings, and aphorisms.

1) Idiom: In A Brief Introduction to the Knowledge of Idioms (1980), Xu Zhaoben believed, "Idioms are phrases (including only a few short sentences) that people have been used for a long time. They are concise in form and incisive in meaning." Modern Chinese Dictionary interprets idiom as "Phrases or word groups that have been used by people for a long time. They are concise in form, incisive in meaning and stereotyped. Chinese idioms are mostly made up of four characters." Idioms are fixed phrases formed by long-term use of the language. Its grammatical function is similar to that of words, but its meaning is richer. Idioms usually have profound ideological connotations, short and incisive, and often with emotional color. Idioms have three features: stereotyped structure, neat syllables, incisive meaning but rich connotations. Most Chinese idioms consist of four characters, but there are also threecharacter idioms and more than four-character idioms. Moreover, some idioms even contain two parts, which are separated by commas. Such as: "an bang ding guo" (stabilize the country), "shang feng bai su" (corrupt morals), "po tian huang" (unprecedented), "bi shang bu zu, bi xia you yv" (less than the top, more than the botto) and so on.

2) Proverb: Wen Dengzheng (2005) defines proverbs as "popular and widely spread stereotyped, concise and visualized sentences, mostly created by working people and reflecting their life experiences and aspirations." Professor Wen divides proverbs into proverbs in a broad sense and proverbs in a narrow sense. Proverb in a broad sense is equivalent to common saying; while the one in a narrow sense is the hyponyms of common saying.

3) Common sayings: Cao Congsun points out in the article A Preliminary Study of Modern Chinese Common Sayings points out that common sayings are popular words spread orally. They are stereotyped sentences with extensive adaptability and complete predicate." Wen Duanzheng (2005) defined the common saying as "a language unit that is created by common people, spread in spoken language, and is colloquial and popular". Moreover, the Chinese Etymologicon revised in 1979 defines common sayings as "established and widely spread stereotyped sentences".

"Common saying" in modern Chinese is called "common verbal saying". For example: "bu jian guan cai bu diao lei." (Do not shed tears until you see the coffin.), "yi bao huan yi bao" (tit for tat), and so on. Common sayings are fixed sentences, which are characterized by popularization and colloquialism. Common sayings are mainly used in spoken language. They are easy to understand, such as "wu shi bu deng san bao dian"(No one comes to the Hall of Trinity without a reason.), "yi ge ba zhang pai bu xiang"(one slap does not make a sound.), "che dao shan qian bi you lu" (There is always a way to go when the car comes to the mountain.) and etc. The Dictionary of Common Sayings (1983) points out that common sayings are popular sayings among people. They are always used by common people, and are also called "colloquial sayings".

4) Two-part allegorical sayings: As a unique type of idiom in Chinese, two part allegorical sayings are authentic language spread in oral form, with vulgar style and humor. There are two types of patterns in two-part allegorical sayings: the complete type and the post-recess type. The complete form is composed of metaphor and explanatory language. The post-recess type can be used when the complete form is familiar to people.

5) Aphorism: Aphorisms are the principles and creeds in words that ancient saints and sages should abide.The style of aphorism is elegant. People often express the 
contents of aphorisms in the form of proverbs, and the elegant style has been diluted with time.

The above classification shows that the classification criteria of Chinese idioms are semantic meaning and pragmatic usage. At the same time, English idioms have similar classification methods. However, it should be noted that Chinese is a paratactic language, and English is a hypotactic one. As a consequence, the classification based on semantics is not widely adopted in English. On the contrary, the classification of idioms based on syntactic structure is more in line with the characteristics of English [5]. We get the following eight categories for English idioms on the basis of syntactic structure:

1) Sentence idioms, such as: Butter wouldn't melt in your mouth.; There's no love between A and B.; Virtue is its own reward.; Your chickens come home to roost.

2) semi-sentence idioms, such as: take the bad with the good, keep a beady eye on sb/sth, beat sb. at their own game, find your bearings.

3) Verbal phrases, such as: have been around, beat up, come across, bring about

4) Prepositional phrases, such as: by the dozen, in doubt, down through, on draught.

5) Nominal idioms, such as: good faith, a golden handshake, hand in glove with sb., an Indian summer

6) Adjective idioms, such as: far and away, full of beans, gone for a burton, further around the road.

7) Words in pairs, such as: aches and pains, safe and sound, give and take, back and forth.

8) fixed similes, such as: flat as a pancake, drunk as a fish, easy as my eyes, poor as a church mouse.

\section{CONCLUSION}

Chinese is a paratactic language emphasizing semantics. However, English emphasizing on syntactic structure is a hypotactic language. The differences are reflected in all levels of language, such as idioms. As a consequence, the definition of Chinese and English idioms is not identical. In addition, as far as the principle of classification of idioms is concerned, syntactic structure is more suitable for English language, though both languages can be classified from the perspective of semantics and pragmatics.

\section{REFERENCES}

[1] Wang You, A Multi-dimensional Contrastive Study on Chinese and English Idioms, in: Doctoral Dissertation, Central China Normal University, May 2015.
[2] Yang Wanjuan, on the species boundary between proverbs and their nearest neighbors, Journal of Central South Institute for nationalities, July 2001.

[3] Yao Xiyuan, the species status and definition domain of" idioms, Chinese character culture, No. 2, 1998.

[4] Collins COBUILD dictionary of English idioms. Shanghai: Shanghai Foreign language Education Press, 2000.

[5] Peng Qinghua, A study of English idioms from a pragmatic Perspective, Beijing: social Science Literature Press, 2007. 